\title{
Percepção ambiental de apicultores: Desafios do atual cenário apícola no interior de São Paulo
}

\author{
Amanda Cerqueiraa ${ }^{*}$; Rodolfo Antônio de Figueiredo ${ }^{a}$ \\ a Programa de Pós-Graduação em Ciências Ambientais, Universidade Federal de São Carlos, São Carlos, São Paulo, 13565-905, Brasil. \\ *ac.cerqueiraamanda@gmail.com
}

Recebido: 20 julho 2017 / Aceito: 24 setembro 2017 / Publicado online: 27 setembro 2017

\begin{abstract}
Resumo
A mortalidade e o desaparecimento de abelhas têm sido cada vez mais frequentes no mundo, afetando consequentemente a atividade apícola que exerce significativa importância nos âmbitos ambiental, social e econômico no Brasil. O objetivo dessa pesquisa foi compreender os principais desafios que a apicultura enfrenta atualmente na perspectiva dos apicultores da região de Matão, interior de São Paulo. Os apicultores foram selecionados por meio da técnica "bola de neve" e participaram da uma entrevista semiestruturada que abordou as esferas social, econômica e ambiental da atividade apícola. A percepção dos apicultores foi analisada através da Análise Textual Discursiva e indicou o atual sistema de produção agrícola, baseado em extensas áreas de monoculturas e uso intensivo de agrotóxicos, como o principal problema enfrentado para manutenção da atividade. Os apicultores percebiam o ambiente considerando o ser humano como parte integrante da natureza, sendo este afetado por suas próprias ações. Tinham a percepção do importante papel das abelhas no equilíbrio do ecossistema e na produção de alimentos. Os relatos apresentados demostram preocupação com a saúde ambiental, enfatizando a falta de comprometimento do governo com a preservação dos recursos naturais e em prol de um sistema de produção agrícola e industrial insustentável.
\end{abstract}

Palavras-chave: apicultura, meio ambiente, produção agrícola, produção de mel.

\section{Environmental perception of beekeepers: Challenges of the current beekeeping scene in the interior of São Paulo}

\begin{abstract}
Mortality and the disappearance of bees have been increasingly frequent in the world, consequently affecting the beekeeping activity that has significant importance in the environmental, social and economic spheres in Brazil. The objective of this research was to understand the main challenges that beekeeping currently faces from the perspective of beekeepers in the region of Matão, in the interior of São Paulo. The beekeepers were selected through the "snowball" technique and participated in a semi-structured interview that addressed the social, economic and environmental spheres of beekeeping. The perception of beekeepers was analyzed through Discursive Textual Analysis and indicated the current system of agricultural production, based on extensive areas of monocultures and intensive use of pesticides, as the main problem faced to maintain the beekeeping activity. The beekeepers perceived the environment considering the human being as an integral part of nature, being affected by their own actions. They were aware of the important role of bees in the balance of the ecosystem and in the production of food. The reports presented demonstrate the real concern with environmental health, emphasizing the government's lack of commitment to the preservation of natural resources in favor of a self-destructive agricultural and industrial production system.
\end{abstract}

Keywords: beekeeping, environment, agricultural production, honey production.

\section{Introdução}

Os serviços ecossistêmicos prestados pelos polinizadores garantem ao ser humano e ao meio ambiente benefícios relativos à produção de alimentos, à conservação da diversidade biológica, à restauração de agroecossistemas e ao crescimento econômico do país (IPBES, 2016; CGEE, 2017). As abelhas são os agentes polinizadores mais eficientes e, junto com os demais polinizadores, são responsáveis por 9,5\% do montante financeiro da produção agrícola de alimentos em nível mundial (Gallai et al., 2009), além da produção de mel e outros produtos na apicultura. Giannini et al. (2015) estimaram os serviços de polinização na produção do campo brasileiro, nas culturas dependentes de polinização, em cerca de U\$ 12 bilhões do total de U\$ 45 bilhões gerados.

Dentre os principais visitantes florais está a abelha africanizada Apis mellifera, que produz cerca de 40 mil toneladas anuais de mel, colocando o Brasil entre os onze maiores produtores mundiais (IBGE, 2016). A apicultura brasileira é responsável por 450 mil trabalhadores no campo, 
além de 16 mil empregos diretos e indiretos, e em 2015 apresentou faturamento de aproximadamente 360 milhões com queda de produção em 1,7\% em relação a 2014 (CBA, 2010; IBGE, 2016). Portanto, é uma atividade agrícola que fornece renda no campo e contribui para a balança comercial no que tange a exportação de alimentos e demais produtos das colmeias (Freitas e Imperatriz-Fonseca, 2005).

Segundo a Intergovernmental Platform on Biodiversity and Ecosystem Services (IPBES), estima-se que haja, aproximadamente, 81 milhões de colméias de abelhas no mundo, responsáveis pela produção de 1,6 milhão de toneladas de mel por ano (IPBES, 2016). Todavia, a produção apícola vem enfretando desafios, que resultam em grandes prejuízos econômicos e ambientais (Gonçalves, 2012), já que a polinização é necessária para a manutenção da flora, em qualquer ecossistema mundial. Logo, interferências negativas sobre os polinizadores podem levar à redução da capacidade reprodutiva das espécies vegetais, afetar a paisagem e a estrutura do ecossistema (Neves, 2008).

Os prejuízos na atividade apícola nos âmbitos econômicosociais e ambientais, podem ser mais significativamente perceptíveis em primeira instância pelos apicultores, uma vez que a produção de mel pode indicar problemas ocorrentes no campo. Marcomin e Sato (2016) discorrem sobre a percepção ambiental considerando os diferentes significados e valores da relação do ser humano com o mundo, considerando as individualidades e a forma de interação de cada sujeito.

Por consequência, tal individualidade traz consigo a dificuldade na percepção da importância da proteção do meio ambiente, já que os diferentes grupos socioeconômicos agem de acordo com a valorização entendida e seus próprios interesses no plano social e ambiental (Hoeffel e Fadini, 2007).

Neste contexto, a presente pesquisa teve por objetivo compreender os principais desafios que a apicultura enfrenta atualmente na perspectiva dos apicultores da região de Matão, interior de São Paulo.

\section{Material e Métodos}

\section{Seleção dos apicultores e delimitação da região de estudo}

A pesquisa foi desenvolvida com 26 apicultores selecionados a partir de uma rede de contatos entre os próprios apicultores, técnica de amostragem denominada snowball sampling (bola de neve), desenvolvida por Goodman (1961) e discutida atualmente no Brasil por pesquisadores como Vinuto (2014). O uso da amostra não probabilística foi necessário perante a dificuldade em localizar os profissionais, uma vez que essa profissão não é, na prática, uma atividade formal, tornando-se um grupo de limitado acesso. Foram encontrados apenas dois registros de apicultores em órgãos públicos da região, sendo esses profissionais as "sementes" que desencadearam as indicações de novos participantes a partir da rede pessoal de cada um.

A "bola de neve" se expandiu em cada entrevista até que o quadro de amostragem se tornou saturado pela falta de indicação que atendesse os critérios da pesquisa, e/ou que não demonstraram interesse em participar do trabalho após a apresentação dos objetivos. O critério de seleção baseou-se na localização onde residem e mantêm os apiários, desenvolvendo assim a atividade apícola na região de Matão, São Paulo.

Tal região se destaca por possuir extensas áreas de cultivo de laranja, atividade agrícola (CITRUSBR, 2017) de alto interesse econômico para agricultores e apicultores, mas sobre a qual se aplica elevada quantidade de agroquímicos, potencialmente tóxicos às abelhas e demais polinizadores, como primeira alternativa de controle a pragas e doenças (Freitas e Pinheiro, 2012; ANVISA, 2017).

\section{Coleta de dados: realização das entrevistas}

As entrevistas foram realizadas entre os meses de janeiro e abril de 2017, em horário e local estabelecidos por cada participante a fim de manter o contato em clima de confiança. A entrevista semiestruturada foi conduzida a partir de 20 questões, mas apenas sete delas referentes à relação "Apicultura x Meio Ambiente" e a relação "Apicultura x Meio Ambiente x Agricultura" (Tabela 1) serão discutidas aqui, além de variáveis sobre o perfil do entrevistado (gênero, faixa etária, tempo de profissão) As gravações foram transcritas e os apicultores participantes serão referidos como "AP1" à "AP26".

Tabela 1. Perguntas realizadas aos apicultores da região de Matão, São Paulo (2017)

\begin{tabular}{l}
\hline \multicolumn{1}{c}{ Assuntos Relacionados/Pergunta } \\
\hline O que as abelhas representam para você? E para o meio \\
ambiente? \\
Sempre manteve os apiários nas mesmas áreas? \\
Nas regiões dos apiários, você presencia a práticas que \\
considera prejudiciais à natureza? \\
\hline \multicolumn{1}{c}{ Agricultura, Apicultura e Meio Ambiente } \\
Quais os principais problemas que enfrenta durante as \\
floradas da cultura de laranja? Há os mesmos problemas em \\
floradas silvestres? \\
Como você relaciona danos ambientais e a agricultura com \\
a atividade apícola?
\end{tabular}

\section{Análise dos dados}

Para análise dos dados foi utilizada a Análise Textual Discursiva, em uma abordagem qualitativa para a compreensão da produção de significados sobre os fenômenos investigados (Moraes e Galiazzi, 2016). A pesquisa, portanto, buscou elementos da profissão que se relacionam intimamente com complexidade do meio ambiente.

\section{Resultados e Discussão}

O perfil dos profissionais apícolas entrevistados foi diversificado quanto a faixa etária (de 26 a 86 anos) e tempo de profissão (de 5 a 52 anos). Em relação ao gênero, a porcentagem de mulheres apicultoras foi de 15,3\% (quatro mulheres), um número aparentemente baixo, mas significativo considerando o empoderamento da mulher frente às profissões majoritariamente masculinas para a sociedade, principalmente 
no que tange o meio rural (Melo e Sabatto, 2008). Neste contexto, faz-se importante ressaltar que o termo "apicultor" aqui fará referência ao profissional apícola, sem distinção de gênero, necessário apenas por uma questão de padronização de tratamento.

A maior parte dos apicultores $(84,6 \%)$ ingressou na profissão a partir de familiares (avôs, pais, tios e primos) e mantiveram-se com as experiências adquiridas e busca de novos conhecimentos. Aqueles que possuem menor tempo de profissão têm a atividade como complemento de renda, manifestando o desejo de dedicarem-se somente a criação de abelhas futuramente. Os que se dedicam a apicultura como profissão integral representam $69,2 \%$ dos entrevistados e, por consequência, são os que dedicam mais tempo ao manejo das colmeias, possibilitando maior produtividade e renda já que a eficiência na produção de produtos apícolas é dependente do objetivo e do manejo que recebem (Senar, 2010).

A partir da questão "o que as abelhas representam para você? E para o meio ambiente?" Se notar que o valor das abelhas vai além do interesse econômico pela venda dos produtos apícolas. Há nas respostas uma dimensão estética, de valor sentimental e de admiração da profissão e do papel das abelhas na produção de alimentos no campo.

Foi citado por três participantes uma frase atribuída a Albert Einstein, que diz que se as abelhas desaparecerem da Terra, a humanidade desaparecerá em quatro anos. Mas, a essência da mensagem foi transmitida de diferentes formas por quase todos os apicultores, como nos seguintes exemplos:

"Para o meio ambiente [a abelha] é tudo! Se não tiver polinização acabou o verde, acabou humano, vida animal" (AP4)

"Já estão falando até na televisão que sem abelha, daqui uns anos, não vai mais ter mundo, né? Vai acabar. Então acho que a abelha é tudo! " (AP7)

"Pro meio ambiente, se a gente perde as abelhas não vai significar mais nada, né? Acabou tudo" (AP14).

Neste sentido, de importância das abelhas ao meio ambiente, faz-se relevante apontar a presença palavra "polinização" nos discursos por significar a percepção de que a importância das abelhas está além da produção de mel. Assim, 50,0\% citam a palavra "polinização" uma ou mais vezes, demostrando conhecimento sobre o serviço ecossistêmico que as abelhas prestam ao meio ambiente e a produção de alimentos no campo; 30,7\% não citam diretamente a palavra "polinização", mas expressam similaridade com polinização abordando questões sobre produção de alimentos, equilíbrio ecossistêmico e importância das abelhas nas floradas enfatizando a produção de mel; e cinco dos $26(19,2 \%)$ apicultores entrevistados consideram que as abelhas são importantes para o meio ambiente, mas não discorrem sobre o porquê dessa importância.

O interesse na migração dos apiários está em conhecer o entorno da área onde eles estão inseridos, a busca por otimização dos recursos alimentares e a predominância da florada que caracteriza o mel. Do total de entrevistados, 76,9\% praticam apicultura migratória e a maioria busca, especificamente, as floradas silvestre, de laranjeira e de eucalipto de acordo com os respectivos meses de floração.

Tal fato demostra que a apicultura migratória é uma alternativa vantajosa à apicultores por não precisarem alimentar as colmeias artificialmente quando não há floradas próximas (Schimer, 1985; Freitas e Imperatriz-Fonseca, 2005). Os apicultores, na busca por recursos florais de culturas agrícolas, almejam uma boa produção de mel e outros subprodutos pela quantidade de pólen e néctar disponíveis às abelhas.

Por outro lado, agricultores são beneficiados pela polinização, garantindo uma produção de frutos e/ou sementes com maior qualidade e quantidade (Schimer, 1985; Freitas e Imperatriz-Fonseca, 2005; Gamito e Malerbo-Souza, 2006), dependendo do nível de dependência da flor pela polinização (Giannini et al., 2015).

A migração em busca da florada de laranjeira está pautada na alta aceitabilidade desse tipo de mel no mercado, nacional e internacional, por apresentar aroma e sabor característicos e ser de coloração mais clara quando comprado aos méis de floradas silvestres e de eucalipto. Porém, a maior rentabilidade pertence ao fruto dessa cultura, uma vez que a as flores de citros quando polinizadas por abelhas aumentam em até $28 \%$ a produção e conferem às frutas características desejáveis pela indústria (Schirmer, 1985; Malerbo-Souza et al., 2004; Gamito e Malerbo-Souza, 2006).

Os benefícios da polinização por A. mellifera em citros, no entanto, parecem não ser relevantes aos produtores da região estudada. As respostas obtidas a partir das perguntas "Quais os principais problemas que enfrenta durante as floradas da cultura da laranja?" e "Nas regiões dos apiários, você presencia práticas que considera prejudiciais à natureza?" apontam o uso de agrotóxicos, aplicado principalmente via pulverização aérea, como sendo o principal responsável por danos ambientais e aos apiários.

Apenas três apicultores $(11,5 \%)$ não mencionaram perdas de colmeias e/ou apiários inteiros pelo menos uma vez durante as floradas, após a aplicação dos agrotóxicos nas monoculturas de laranja e cana-de-açúcar. Do total de participantes, $88,5 \%$ dos apicultores relataram perdas significativas de colmeias e/ou apiários inteiros ocasionada por agrotóxicos. Os efeitos notados após a pulverização são grande quantidade de abelhas mortas próximas as colmeias e/ou abelhas desorientadas, colmeias que deixam de produzir ou não conseguem se fortalecer para produzir, abandono das crias, presença de muitos insetos mortos próximo aos apiários e cheiro de veneno. Apontaram ainda que muitas vezes a pulverização é realizada sem a devida fiscalização e controle das condições climáticas.

O uso indiscriminado de agrotóxicos tem causado impacto sobre a população de abelhas em todo o mundo (Freitas; Pinheiro, 2012; Marques et al., 2015; Gallai et al., 2009). Os autores Marques et al. (2015) e Pacífico da Silva et al. (2016) enfatizam ainda que a disseminação desses produtos muitas vezes não vem acompanhada pela divulgação dos efeitos adversos à saúde do meio ambiente e dos seres vivos.

O Instituto Nacional do Câncer (INCA, 2017) traz que em 2009 o Brasil se tornou líder mundial no consumo de agrotóxicos, fato impulsionado pelo incentivo do governo 
brasileiro a partir da redução dos impostos e à liberdade de utilização de princípios ativos banidos em outros países, conforme pode-se constatar na lei $\mathrm{n}^{\mathrm{o}} .7 .802 / 89$, no Decreto n. $8133 / 13$ e na lista de ingredientes ativos com uso autorizado e banidos no Brasil da Anvisa (2017).

Documento do IPBES (2016) cita que a Convention on Biological Diversity (CBD) sugere maior avaliação dos riscos a polinizadores e divulgação ao público de alguns pesticidas. Ação importante, pois, os agrotóxicos atualmente utilizados, tais como os neonicotinoides, são altamente tóxicos às abelhas, afetando a memória, o olfato, o voo e a orientação (Gonçalves, 2012; Fairbrother et al., 2014).

Além do uso de agrotóxico, a expansão das áreas de monocultura de cana-de-açúcar na região, com consequente perda de habitat, está entre os fatores mais citados como prejudiciais à apicultura e ao meio ambiente. A produção de cana-de-açúcar no estado de São Paulo é responsável por $55,2 \%$ da produção nacional. Essa cultura apresentou crescimento de 6,2\% de área plantada em 2016 em São Paulo, totalizando 4.777,2 mil hectares (CONAB, 2016). O CONAB (2016) enfatiza ainda que as condições climáticas foram favoráveis em São Paulo e contribuíram para o desenvolvimento dos canaviais recém-plantados. Tais dados confirmam dos relatos dos apicultores sobre a expansão das áreas plantadas de cana-de-açúcar na região estudada.

A questão que tratava sobre a relação dos danos ambientais e a agricultura com a atividade apícola, levantou algumas críticas pelos apicultores em relação a falta de empenho do governo para se combater o uso de agrotóxicos potencialmente tóxicos aos polinizadores, ao solo e à água, ao mesmo tempo em que trazem o questionamento sobre os prejuízos para a saúde humana a partir da ingestão de veneno.

A crítica ao governo se estende a falta de incentivo ao desenvolvimento da apicultura no Brasil e o não reconhecimento da profissão. Apenas 7,5\% dos participantes citam o Programa Nacional de Fortalecimento da Agricultura Familiar (Pronaf) como sendo uma ação governamental que beneficia o apicultor, e $11,5 \%$ reconhecem o Serviço Brasileiro de Apoio às Micro e Pequenas Empresas (SEBRAE) como órgão engajado no desenvolvimento da apicultura.

A maior parte dos entrevistados $(80,7 \%)$, considera que não recebe apoio de qualquer tipo de organização (governamental ou não) no trabalho com as abelhas, e alguns ressaltam a alta taxa de impostos sobre a venda de mel. Sobre isso, destaca-se a seguinte fala:

"Você tem o melhor produto do mundo, uns dos melhores méis do mundo. Nosso mel é todo exportado, todos lá for compram mel e aqui você não tem apoio do governo, né? Aqui nosso apoio infelizmente é zero" (AP6).

Assim, em localidades onde o apicultor é inserido no mercado formal, geralmente há o entendimento do papel da apicultura naquela região, considerando sua importância econômica e nos serviços ecossistêmicos (PASSIN; TERESO, 2008), fato que não condiz com o atual cenário apícola brasileiro, segundo os relatos dos apicultores entrevistados.

\section{Conclusões}

Os apicultores entrevistados possuem percepção ambiental crítica diante dos prejuízos e danos ambientais ocasionados pelas ações antrópicas que afetam intimamente a profissão. Há preocupação frente a saúde ambiental e ao futuro da produção agrícola pelo declínio de polinizadores, em especial às abelhas. Percebem que os danos ambientais ocasionados pelo atual modelo agrícola brasileiro, pautado em extensas áreas de monoculturas e aplicação de produtos químicos potencialmente tóxicos ao meio ambiente, afeta diretamente os apicultores por serem, em sua maioria, profissionais autônomos dependentes de pasto apícola cada vez mais reduzido e menos disponível às abelhas melíferas.

Há o entendimento por parte dos apicultores sobre a importância das abelhas à produção de alimentos no campo e na reprodução das espécies vegetais, sendo a produção de mel expressiva para a economia do país. As informações obtidas nesta pesquisa mostram que ações de políticas públicas e de iniciativas privadas/industriais frente aos desafios apontados são urgentes e necessárias não só ao setor apícola, mas também visando a saúde ambiental, de produção de alimentos e segurança alimentar.

\section{Agradecimentos}

Ao Departamento de Ciências Ambientais da Universidade Federal de São Carlos pelo incentivo a pesquisa, e à Coordenação de Aperfeiçoamento de Pessoal de Nível Superior (CAPES) pelo apoio financeiro à primeira autora. Agradecemos em especial aos apicultores que aceitaram participar das entrevistas, sem os quais não seria possível o desenvolvimento deste artigo.

\section{Referências}

ANVISA - Agência Nacional de Vigilância Sanitária. Listas de ingredientes ativos com uso autorizado e banidos no brasil. Acesso em 17/07/2017.

Brasil. 2013. Decreto ${ }^{\circ}$ 8.133, de 28 de outubro de 2013. Acesso em 20 set. 2017.

Brasil. 1989. Lei n 7.802 , de 11 de julho de 1989 . Acesso em 20 set. 2017. CBA. 2010. Confederação brasileira de apicultura acesso em 01 jun. 2017.

CGEE - Centro de gestão e estudos estratégicos. 2017. Importância dos polinizadores na produção de alimentos e na segurança alimentar global. Brasília, DF: CGEE. 124p. Acesso em 08 jul. 2017.

CITRUSBR. 2017. Associação nacional dos exportadores de sucos cítricos acesso em 20 jul. 2017.

CONAB - Companhia Nacional de Abastecimento. 2016. Monitoramento agrícola - cana-de-açúcar. Acompanhamento da safra brasileira: cana-deaçúcar, v. 3 - safra 2016/17, n. 3, brasília, p. 1-74. Acesso em 22 set. 2017.

Fairbrother, A.; Purdy, J.; Anderson, T.; Fellk, R. 2014. Risks of neonicotinoid insecticides to honeybees. Environmental toxicology and chemistry, 33(4): 719-731.

Freitas, B.M.; Imperatriz-Fonseca, V.L. 2005. A importância econômica da polinização. Mensagem doce, 80: 44-46. Acesso em 19 set. 2017.

Freitas, B.M.; Pinheiro, j.n. 2012. Polinizadores e pesticidas: princípios e manejo para os agroecossistemas brasileiros. MMA, brasília, 112p. Acesso em 22 jul. 2017.

Gallai, N.; Salles, J.M.; Settele, J.; Vaissière, B.E. 2009. Economic valuation of the vulnerability of world agriculture confronted with pollinator decline. Ecological economics, 68: 810-821.

Gamito, L.M.; Malerbo-Souza, d.t. 2006. Visitantes florais e produção de frutos em cultura de laranja (citrus sinensis 1. Osbeck). Acta Scientiarum Biological sciences, 28(4): 483-488.

Giannini, T.C.; Cordeiro, G.D.; Freitas, B.M.; Saraiva, A.M.; ImperatrizFonseca, V.L. 2015. The dependence of crops for pollinators and the economic value of pollination in brazil. Journal of economic entomology, 108: $1-9$.

Gonçalves, L.S. 2012. O desaparecimento das abelhas, suas causas, consequências e o risco dos neonicotinóides para o agronegócio apícola. Mensagem doce, 117. Acesso em 15 jul. 2017.

Goodman, L. 1961. Snowball sampling. Annals of mathematical statistics, v. 32, p. 148-170 acesso em 19 jul. 2017 
Hoeffel, J.L.; Fadini, A.A.B. 2007. Percepção ambiental. In: ferraro jr., 1.a. (org). Encontros e caminhos: formação de educadoras ambientais e coletivos educadores. V.2. Ministério do meio ambiente, brasília, p. 255262. Acesso em 21 set. 2017.

IBGE - Instituto Brasileiro de Geografia e Estatística. 2016. Produção da pecuária municipal. Rio de janeiro, 43, p. 1-49. Acesso em 22 set. 2017.

INCA - Instituto Nacional do Câncer. 2015. Brasil lidera o ranking de consumo de agrotóxicos. Rio de janeiro: ministério da saúde. Acesso em 17 jul. 2017.

IPBES - Intergovernmental Science-Policy Platform on Biodiversity and Ecosystem Services. 2016. Summary for policymakers of the assessment report of the intergovernmental science-policy platform on biodiversity and ecosystem services on pollinators, pollination and food production. Bonn, germany: secretariat of the intergovernmental science-policy platform on biodiversity and ecosystem services. 36p. Acesso em 18 jul. 2017.

Malerbo-Souza, D.T.; Nogueira-Couto, R.H.; Couto, L.A. 2004. Honey bee attractants and pollination in sweet orange, citrus sinensis (1.) Osbeck, var. Pera-rio. Journal of Venomous Animals and Toxins Including Tropical Diseases, 10(2): 144-153.

Marcomin, F.E.; Sato, M. 2016. Percepção, paisagem e educação ambiental: uma investigação na região litorânea de Laguna-SC, brasil. Educação em revista, 32(2): 159-186.

Marques, M.F.; Menezes, G.B.; Deprá, M.S.; Delaqua, G.C.G.; Hautequestt, A.P.; Moraes, M.C.M. 2015. Polinizadores na agricultura: ênfase em abelhas. Funbio, Rio de Janeiro. Acesso em 22 set. 2017.

Melo, H.P.; Sabbato, A.D. 2008. Censo da reforma agrária de 1996 e 1997 em uma perspectiva de gênero. In: Lope, A.L.; Butto, A. (orgs.) Mulheres na reforma agrária. A experiência recente no brasil. MDA, Brasília, p.39-80. Acesso em 20 ago. 2017.

Moraes, R.; Galiazzi, M.C. 2016. Análise textual discursiva. 3a ed. UNIJUI, Ijuí, 224p.

Neves, E.L. 2008. Polinização de espécies nativas da caatinga e o papel da abelha exótica apis mellifera 1. Tese de doutorado, Universidade Estadual de Feira de Santana, Feira de Santana, Bahia. 155p. Acesso em 18 set. 2017.

Pacífico da Silva, I.; Melo, M.M.; Soto-Blanco, B. 2016. Efeitos tóxicos dos praguicidas para abelhas. Revista brasileira de higiene e sanidade animal, 10(1): 142-157.

Pasin, L.E.V.; Tereso, M.J.A. 2008. Análise da infra-estrutura existente em unidades de produção agrícola para processamento de mel na região do vale do paraíba-sp. Ciência e agrotecnologia, 32(2): 510-516.

Schirmer, L.R. 1985. Abelhas ecológicas. Nobel, são paulo. 218p.

Senar. 2010. Serviço nacional de aprendizagem rural. Abelhas apis mellifera: instalação do apiário. Serviço nacional de aprendizagem rural, 2. Ed. Brasília.

Vinuto, J. 2014. A amostragem em bola de neve na pesquisa qualitativa: um debate em aberto. Temáticas, 44: 203-220. 